\title{
Virtual Reality and Its Application in the Study of Dust Diffusion
}

\author{
Tonghui Zhao ${ }^{1) 2)}$ Zhen Zhou $^{1)}$ Tianling Lv ${ }^{2)}$ Rui Zhao ${ }^{2)}$ \\ 1)( School of Measurement and Communication, Harbin University of Science \& Technology \\ Harbin 150080) \\ 2)( Labor Safety Science and Technology Research Center of Heilongjiang Province \\ Harbin 150001)
}

Key words: Virtual Reality; Dust spread; Simulation

\begin{abstract}
Virtual reality, a new technology in the world, is introduced and discussed according tothe regularity of dust dispersion in the atmosphere, and on the basis of mathematical model, a three dimensional dynamic scene of dust dispersion is developed by adopting OpenGL, inwhich the parameters of flow field and observation view angle could precisely be adjusted.The same 3- D scene under the impact of gravity and wind speed etc, and under the reactionof jet - flow may also be establushed. This method provides scientific criteria for wellhandling the regularity of dust dispersion, and searching rationalpreventive measures. Thistechnology also has good appliction prospects in other field of safety science.
\end{abstract}

\section{Preface}

Virtual Reality (V R), the use of computer graphics, multimedia, electronics, networking, a variety of sensors and other high-tech to construct a virtual realm ${ }^{[3]}$. People can interact with this environment through a variety of sensing systems or information input devices to achieve the same sense of reality ${ }^{[4]}$. Virtual reality has become a topic of international concern, a variety of hardware and software manufacturers competing to provide a variety of commercial products.Although the domestic technology for the prevention of safety and occupational hazards has just started, the development of fast, compared with foreign countries, there is still a big gap, is still in the "application" and "follow" stage ${ }^{[5]}$.

Fire, explosion and poison gas leakage and other accidents often cause a lot of casualties, and dust is an important factor leading to occupational hazards.It is an important way to prevent accidents from casualties and control occupational hazards by predicting the extent of the accident and investigating the distribution of dust in the operating environment.Virtual technology can construct a three-dimensional dynamic scene of accident scene and dust distribution. In terms of dust hazards, the spread of dust in the study environment can provide a basis for people to develop reasonable and effective prevention and control measures.

The author's paper discusses virtual reality technology and its implementation tools. At the same time, based on the dust diffusion model in the environment, the virtual reality of the three dimensional scene of dust in the air source is constructed.

\section{2 . Virtual Reality Classification And Implementation Tools}

Virtual reality technology is the latest and highest level of simulation technology. Real-time, interactive three-dimensional environment, coupled with immersion, so that virtual reality can be reconstructed around the real world.

\section{1 Type of Virtual Reality}

According to the use of the software environment, virtual reality can be divided into single and network type. Single type only create a scene on the machine to establish the scene for the use of 
the machine; network type in the server to establish a scene for other computers using specific customer software through the network to access.

Support users can be divided into multi-user type and single-user type. Multi-user applications for entertainment, chat rooms, virtual communities, remote (education, training, conferences, surgery, etc.) and specialized projects (such as military simulation); Single-user applications for e-commerce, website construction and specialized projects (such as real estate demonstrations, driving training, scientific visualization, commercial data visualization, engineering design, etc.). According to immersion of the system and hardware to achieve classification, it can be divided into desktop V R system and immersion VR system, and immersion V R system can be divided into: helmet-mounted display, immersive projection display and cockpit V R system.

Desktop A system refers to a window that views one or more computer screens as participants in a virtual environment, with the usual keyboard, mouse, joystick or traditional VR interactive tools: six degrees of freedom tracking ball, data gloves, head trackers as input devices, with simple shutter glasses to create three-dimensional visual fruit, increase the degree of immersion. But the participants can still see the surrounding real environment, immersion is not strong, suitable for simple V R application system. Immersive VR system refers to the computer system and special specially dedicated sensor connected to the same time with a variety of output devices and human interaction, people get the same feeling with the real world.

\section{2 implementation tools}

The tools to implement virtual reality are related to their type and function, and the commonly used tools are divided into three categories:

1) three-dimensional animation class: in the machine to build a schematic three-dimensional scene, the effect of it is realistic, simple production, but the machine requirements are high, it can't be precisely controlled, such as 3DM AX, Maya.

2) Network scene class: it is achieved on the server. Network users are accessed through the client program, especially for the application on the Internet. The local requirements is low, network transmission of information is less, but lack of control flexibility, such as V RM L, Java3D.

3) Direct control class: when the establishment of scientific scenes, the requirements of the object involved in the flexible, precise control, such as OpenGL, Direct3D, but programming more difficult.

In practice, the right tool can be selected according to the site requirements.

\section{Dust Diffusion Model}

The diffusion model of the source dust in the atmosphere is the core of the virtual scene. The diffusion process is affected by the airflow state, the physical and chemical properties of the dust and the aerosol properties of the particles. Because of the large number of factors and the complexity of the model, the model is often based on the assumption that in the isotropic matter, the diffusion is in accordance with the law of Fischer, that is, the velocity of the diffusion material passing through the unit area is proportional to the concentration gradient of the surface ${ }^{[1]}$

$$
\mathrm{F}=-\mathrm{D} \frac{\partial \mathrm{C}}{\partial \mathrm{x}}(1)
$$

$$
\frac{\partial \mathrm{C}}{\partial \mathrm{t}}=D\left[\frac{\partial^{2} \mathrm{C}}{\partial \mathrm{x}^{2}}+\frac{\partial^{2} \mathrm{C}}{\partial y^{2}}+\frac{\partial^{2} \mathrm{C}}{\partial z^{2}}\right]
$$

Turbulence is the state where the air is often in. The diffusion of pollutants in turbulence usually conforms to certain statistical laws. The turbulence statistical method is combined with Fick's law to obtain the following model:

1) For a continuous homogeneous point source located at the origin of the coordinate ${ }^{[2]}$, the concentration on the $\mathrm{y}$ and $\mathrm{z}$ axes coincides with the normal distribution in the $\mathrm{x}$ direction uniform wind speed $\mathrm{u}$, the spatial concentration $(\mathrm{x}, \mathrm{y}, \mathrm{z})$ of the dust concentration is: 


$$
\mathrm{C}(\mathrm{x}, \mathrm{y}, \mathrm{z})=\frac{q}{2^{c^{e^{e}} y^{e_{z}}}} \exp \left[-\left(\frac{y^{2}}{2^{e_{y}^{2}}}+\frac{z^{2}}{2^{e_{z}^{2}}}\right)\right](3)
$$

q - source strong, unit g / s;

$e_{y}, e_{z}$ - the standard deviation of the pollutant distribution in the $y, z$ direction, unit: $m$, its value and the stability of the air flow, experimental and empirical data can be investigated.

2) When considering the point source from the ground has a certain height $\mathrm{H}$, and the ground does not reflect the role of dust:

$$
\mathrm{C}(\mathrm{x}, \mathrm{y}, \mathrm{z}, \mathrm{H})=\frac{q}{2^{c_{u} e_{y} e_{\mathrm{z}}}} \exp \left[-\left(\frac{y^{2}}{2^{e_{y}^{2}}}+\frac{(z-H)^{2}}{2^{e_{\mathrm{z}}^{2}}}\right)\right](4)
$$

3) Dust particles and gaseous pollutants are different, especially when the particle size $>10^{\mu}$, the role of gravity sedimentation can not be ignored:

$$
\mathrm{C}(\mathrm{x}, \mathrm{y}, \mathrm{z}, \mathrm{H})=\frac{q}{2^{c_{u} e_{y} e_{z}}} \exp \left[-\left(\frac{y^{2}}{2^{e_{y}^{2}}}+\frac{\left(z-\left(H-v_{t} x / u\right)\right)^{2}}{2^{e_{z}^{2}}}\right)\right](5)
$$

The particle settling velocity $\mathrm{v}_{\mathrm{t}}$ can be given by the following formula:

$$
\mathrm{v}_{t}=\frac{\left(d_{p}-d_{g}\right) g}{18} d_{p}^{2}(6)
$$

$\mathrm{d}_{\mathrm{p}}, \mathrm{d}_{\mathrm{g}}$ - settable particles and air density, $\mathrm{kg} / \mathrm{m}^{3} ; \mathrm{d}_{\mathrm{p}}$ - sediment particle diameter, $\mathrm{m}_{\text {; }}$ - - air viscosity, $\mathrm{kg} /(\mathrm{m} \cdot \mathrm{s})$.

\section{Realization of Dust Diffusion Virtual Scene}

Aiming at the process of diffusion of dust in the atmosphere, based on the mathematical model, the desktop V R system was used to construct the three-dimensional scene of the dust particles in the air flow. Users interact with the system through the keyboard, mouse, monitor and other standard input and output devices to interact with the system.Fig1 and Fig 2 are views without wind conditions and Fig3 and Fig 4 are views under uniform wind speed conditions. Fig 5 、Fig6、Fig7、 Fig 8 shows the views of the dust produced in the direction of gravity (along the $\mathrm{X}$ direction) and the lateral flow (along the $\mathrm{Z}$ direction) in several directions..

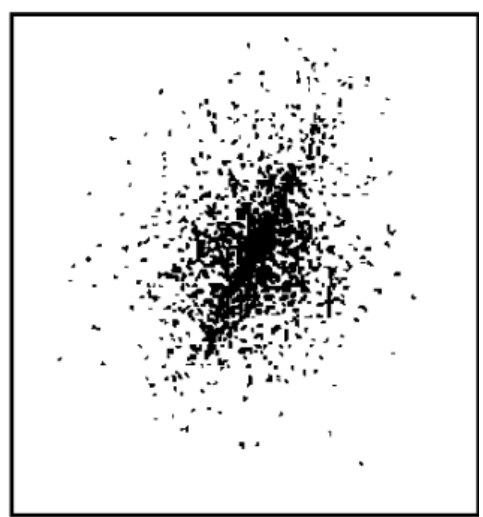

Fig. 1 point source without wind does not consider the impact of gravity 


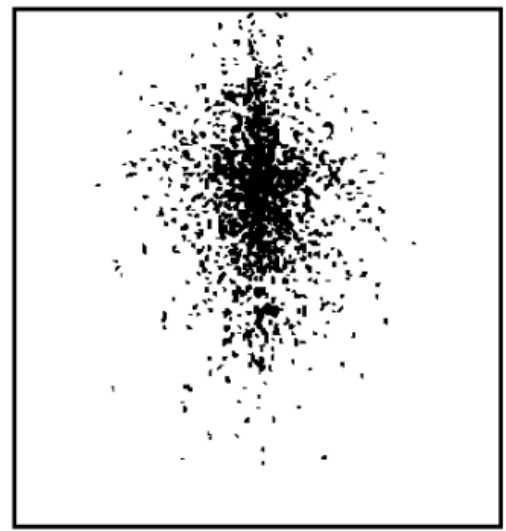

Fig. 2 Point source without wind Consider gravity

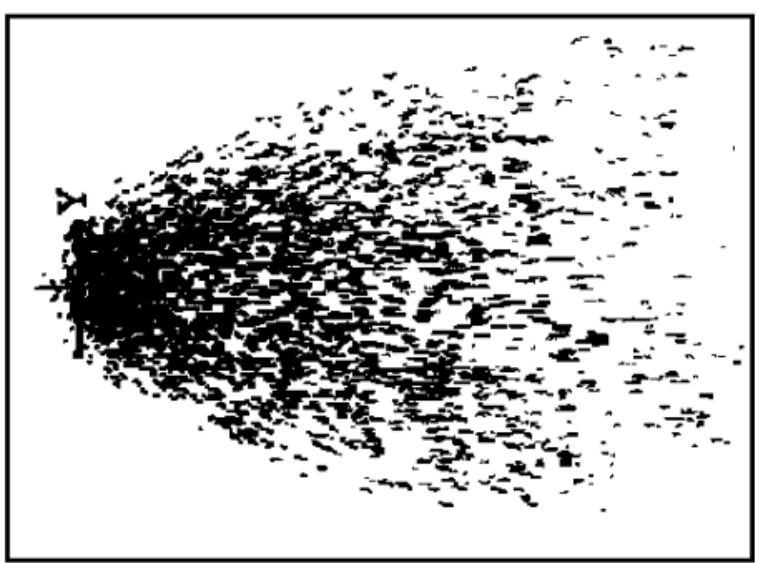

Fig. 3 point source uniform wind speed does not consider the impact of gravity

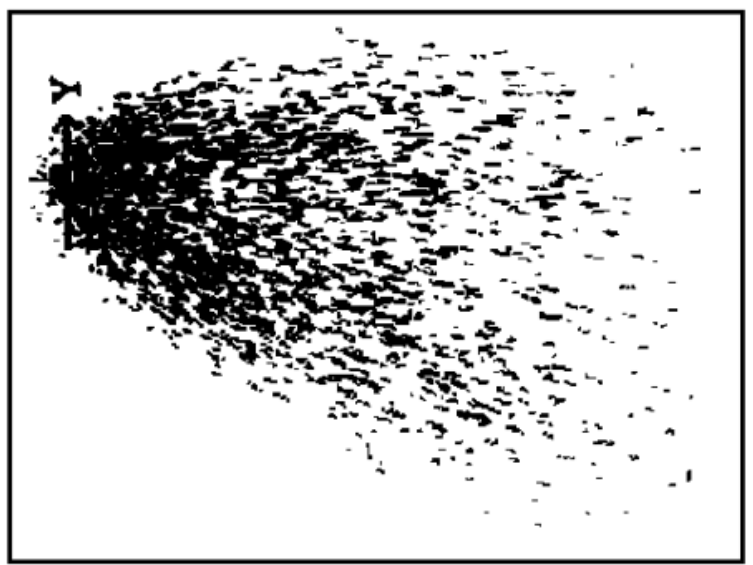

Fig. 4 point source $x$ to the uniform wind speed to consider the impact of gravity

1) Due to the establishment of the scene to scientific computing and particle system, the scene using OpenGL development, programming language for the $\mathrm{V}$ C. Although the workload of programming and very difficult, flexibility and precise control has achieved, showing a real diffusion process.

2)The establishment of the virtual reality is a three-dimensional scene, using OpenGL perspective transformation technology, through the keyboard and mouse drag the way to the scene three-dimensional rotation, movement and zoom and other operations, so that people from different perspectives to observe the dust particles in the air diffusion process. 
3) The established scene is based on the model, taking full account of the effect of airflow speed, jet velocity and dust particle weight on diffusion. When the value of the parameter changes, the virtual scene can be changed accordingly.

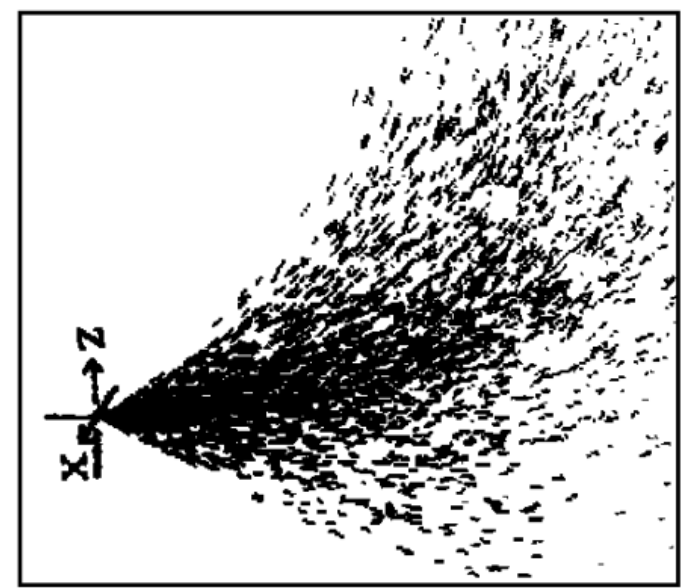

Fig. 5 gravity (X direction) and lateral flow (Z direction) under the action of jet dust distribution

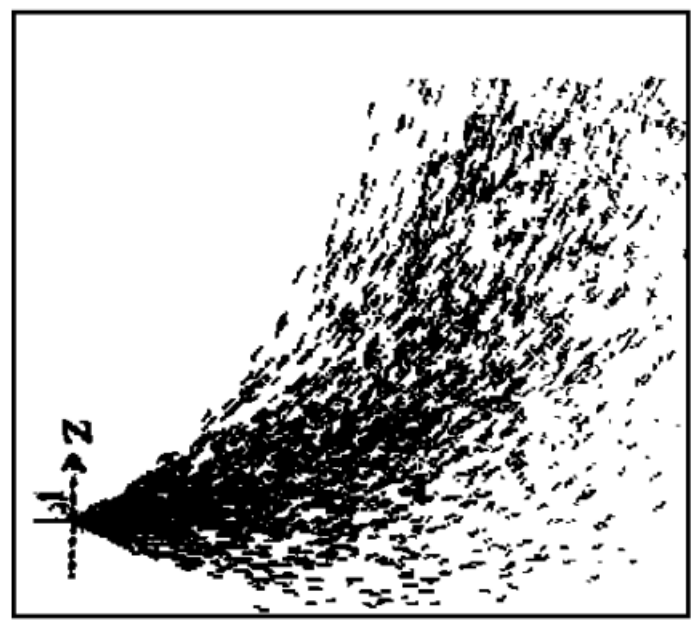

Fig. 6 Z-direction lateral flow effects

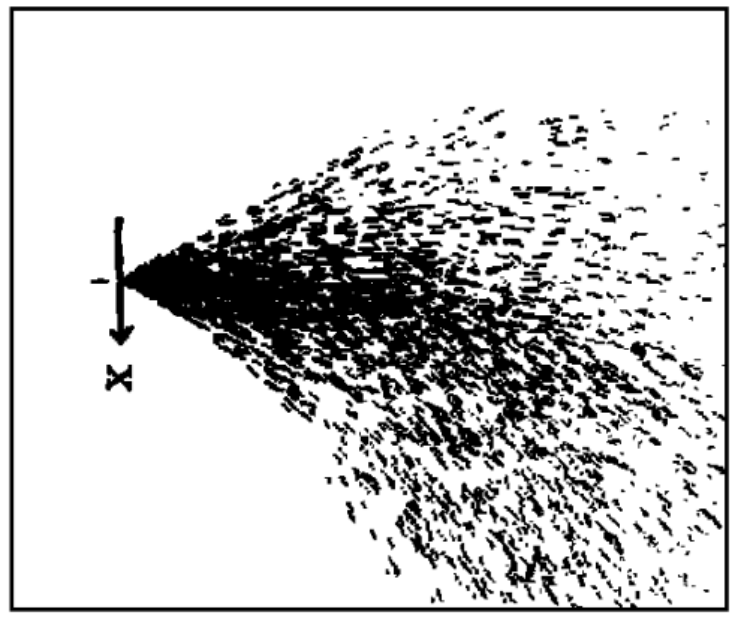

Fig. $7 \mathrm{X}$ gravity effect 


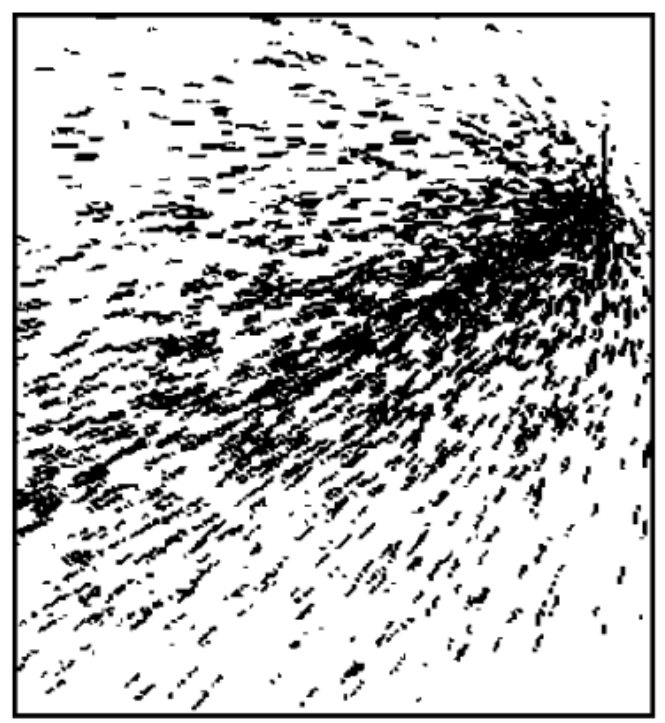

Fig. 8 Effect of X-direction gravity and Z-direction lateral flow

4) Particle system programming has its particularity, in the establishment of three-dimensional particle injection scene, the particle life cycle, particle starting color, jet ejection angle adjustable, so that the establishment of the system more in line with the real situation.

Associating the sensor and output device with the user, the real feelings of dust in the three-dimensional space diffusion can be obtained.

\section{5 . Conclusion}

The author discusses the virtual reality technology and uses it to study the diffusion of dust in the atmosphere. Based on the mathematical model of diffusion, the three-dimensional dynamic scene of the diffusion process is established. Virtual reality is a very promising technology, especially in the field of security can complete the special equipment operation training, accident process simulation, emergency escape drill, safety education, fire and air leakage and spill movement simulation, equipment safety design tasks. In other areas also have broad application prospects.

\section{References}

[1] Quanquan Zhang edited. Aerosol mechanics - Theoretical basis for dust removal. Beijing: China Environmental Science Press, 1987. 12.

[2] Enqi Pu edited. Air Pollution Control Project. Beijing: Higher Education Press, 1999. 6.

[3] Fenfang Zeng edited. Virtual Reality Technology. Shanghai Jiaotong University Press, 1997. 6.

[4] John N. Latta a nd Dav id J. O berg , A Conceptua I Vir tual Reality M odel, Co mputer Graphics andApplica tio ns, 1994, 14( 1): 23-29.

[5]David Kahaner , Japanese Activities in V ir tual Reality , Co mputer G raphics and Applicatio ns, 1994, 14( 1): 75-78. 